\title{
Measurements of Charge Transfer Inefficiency in a CCD with High-Speed Column Parallel Readout
}

\author{
André Sopczak ${ }^{1}$, Khaled Bekhouche ${ }^{1}$, Chris Damerell ${ }^{2}$, \\ Tim Greenshaw ${ }^{3}$, Michal Koziel ${ }^{1}$, Konstantin Stefanov ${ }^{2}$, \\ Tuomo Tikkanen ${ }^{3}$, Tim Woolliscroft ${ }^{3}$, Steve Worm ${ }^{2}$ \\ ${ }^{1}$ Lancaster University, UK \\ ${ }^{2}$ STFC Rutherford Appleton Laboratory, UK \\ ${ }^{3}$ Liverpool University, UK
}

\begin{abstract}
Charge Coupled Devices (CCDs) have been successfully used in several high energy physics experiments over the past two decades. Their high spatial resolution and thin sensitive layers make them an excellent tool for studying short-lived particles. The Linear Collider Flavour Identification (LCFI) collaboration is developing Column-Parallel CCDs (CPCCDs) for the vertex detector of a future Linear Collider. The CPCCDs can be read out many times faster than standard CCDs, significantly increasing their operating speed. A test stand for measuring the charge transfer inefficiency (CTI) of a prototype CPCCD has been set up. Studies of the CTI have been performed at a range of readout frequencies and operating temperatures.
\end{abstract}

Presented on behalf of the LCFI Collaboration at the IEEE 2008 Nuclear Science Symposium, Dresden, Germany 



\title{
Measurements of Charge Transfer Inefficiency in a CCD with High-Speed Column Parallel Readout
}

\author{
André Sopczak, Member, IEEE, Khaled Bekhouche, Chris Damerell, Tim Greenshaw, Michal Koziel, \\ Konstantin Stefanov, Tuomo Tikkanen, Tim Woolliscroft, Steve Worm
}

\begin{abstract}
Charge Coupled Devices (CCDs) have been successfully used in several high energy physics experiments over the past two decades. Their high spatial resolution and thin sensitive layers make them an excellent tool for studying shortlived particles. The Linear Collider Flavour Identification (LCFI) collaboration is developing Column-Parallel CCDs (CPCCDs) for the vertex detector of a future Linear Collider. The CPCCDs can be read out many times faster than standard CCDs, significantly increasing their operating speed. A test stand for measuring the charge transfer inefficiency (CTI) of a prototype CPCCD has been set up. Studies of the CTI have been performed at a range of readout frequencies and operating temperatures.
\end{abstract}

\section{INTRODUCTION}

The study of radiation hardness is crucial for the application of CCD detectors in High Energy Physics experiments [1]-[3]. The LCFI collaboration has been developing and testing new CCD detectors for about 10 years [1]-[5]. Previous experimental results on CCD radiation hardness were reported for example in [6]-[8]. Several models increased the understanding of radiation damage effects in CCDs [9]-[11]. The measurements and analyses reported in this paper have been carried out in the LCFI collaboration [3]. Simulation and modelling of CCD radiation hardness effects for a CCD prototype with sequential readout was reported at IEEE'2005 [12], comparing full TCAD simulations with analytic models was reported at IEEE'2006 [13] and in Ref. [14], simulation and modelling of a CCD prototype with column parallel readout (CPCCD) at IEEE'2007 [15] and in Ref. [16].

This work focuses on experimental measurements and a method to determine the charge transfer inefficiency (CTI) performed with the CPCCD at a test stand at Liverpool University.

The environment with high radiation near the interaction point at a future Linear Collider creates damage to the CCD material which leads to defects acting as electron traps in the silicon, as indicated in Fig. 1. The mechanism of creating traps has been discussed in the literature, for example in Refs. [17][19]. These traps result in charge transfer inefficiency. In a

A. Sopczak is with Lancaster University, UK. Presented on behalf of the

LCFI Collaboration; E-mail: andre.sopczak@cern.ch

$\mathrm{K}$. Bekhouche is with Lancaster University, UK

C. Damerell is with STFC Rutherford Appleton Laboratory, UK

T. Greenshaw is with Liverpool University, UK

M. Koziel is with Lancaster University, UK

K. Stefanov is with STFC Rutherford Appleton Laboratory, UK

T. Tikkanen is with Liverpool University, UK (currently with Leicester

University, UK)

T. Woolliscroft is with Liverpool University, UK

$\mathrm{S}$. Worm is with STFC Rutherford Appleton Laboratory, UK

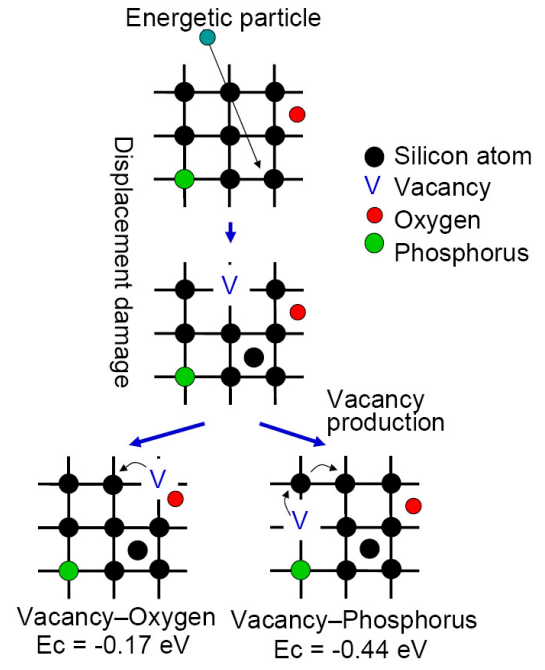

Fig. 1. Schematic view of how radiation damage is created in phosphorusdoped silicon. Two types of vacancy defect are created: Vacancy-Oxygen (electron trap at $0.17 \mathrm{eV}$ below the conduction band) and Vacancy-Phosphorus (electron trap at $0.44 \mathrm{eV}$ below the conduction band).

phosphorus-doped device, two types of traps are created. The first one is relatively shallow with energy level $0.17 \mathrm{eV}$ below the conduction band and the second is deep with energy level $0.44 \mathrm{eV}$ below the conduction band. These traps result in charge transfer inefficiency.

The column parallel technology is in development to cope with the required readout rate. CPC1 is a two-phase CCD prototype capable of $50 \mathrm{MHz}$ readout frequency. In this paper we demonstrate the method to determine the CTI value with an un-irradiated CPCCD (CPC1).

\section{TEST STAND FOR CCD OPERATION AND CRYOSTAT UNIT}

A test stand has been set up with readout electronics and a cryostat unit. The temperature range of the cryostat is from room temperature down to about $-140{ }^{\circ} \mathrm{C}$. This temperature has been achieved with cold nitrogen gas by boiling liquid nitrogen. The very low operating temperature is required to obtain sensitivity to the CTI peak structure for $0.17 \mathrm{eV}$ traps where the peak position is expected to be near $-130{ }^{\circ} \mathrm{C}$ [16]. Initial measurements have been performed on an un-irradiated device in standalone mode, where the signal from four columns of the CCD were amplified and connected to external ADCs. An ${ }^{55} \mathrm{Fe}$ source emitting $6 \mathrm{keV}$ X-rays was attached to a holder at a distance of $5 \mathrm{~cm}$ from the CCD to provide the signal charge. Figure 2 shows a picture of $\mathrm{CPC} 1$ 


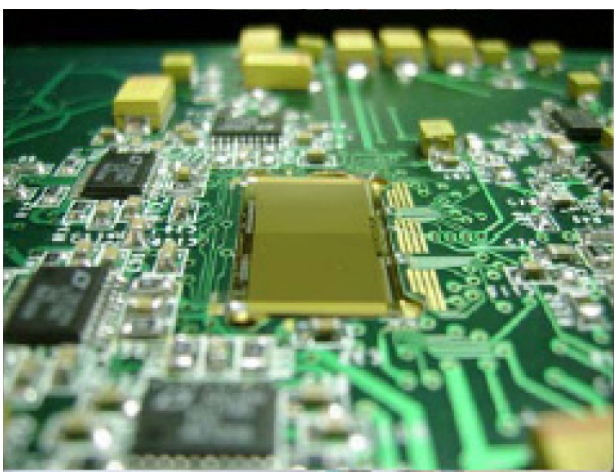

Fig. 2. CPC1 (in the center of the picture) with external electronics.

and the associated control and front-end readout electronics, which were also placed in the cryostat, wherefrom the four outputs were fed to rack-mounted amplifiers and ADCs.

It was observed that the CTI increased sharply when the amplitude of the sinusoidal clock pulses applied to read out the $\mathrm{CCD}$ was reduced to less than $2 \mathrm{~V}$ peak-to-peak. In order to suppress any effect from the clock amplitude, settings for each data acquisition were tuned to produce $3.00 \mathrm{~V}_{\mathrm{pp}}$ clock pulses. The performance of the drivers limited the maximum clocking frequency to about $22 \mathrm{MHz}$. The settings depended on the frequency and had to be adjusted for each cryostat temperature.

\section{Signal MEASuREMEnt AND Removing Charge SHARING BY USING A $3 \times 3$ CLUSTER METHOD}

The fast ADCs convert the signal charge after amplification with a wideband preamplifier. The four columns are read out in 4 channels by ADCs. Three columns (channels 1 to 3) are adjacent whereas channel 4 is separate. The signal charges of 400 pixels plus 10 overclocks were acquired in 3000-5000 frames per measurement. This leads to an accurate statistical precision. The collected data has been analysed using the ROOT package [20]. First, we begin by applying correlated double sampling, where the difference between the signals of two consecutive pixels is taken to be the signal charge collected by the latter pixel. This decreases electronic noise. Next, we remove common mode noise components for each column with the help of the overclocks. Overclocks are charges read out after the last pixel of the column. We average all overclocks of a column and subtract the average from the data of all pixels in the column. As an example for channel 2 Fig. 3 shows the distribution of ADC codes for all recorded pixels and all frames of one measurement. The analysis is performed on column 2 because it is situated between columns 1 and 3 . This allows to apply a $3 \times 3$ cluster method.

Figure 3 illustrates that there are 3 regions in ADC codes. The first region (I) has a high number of pixels with low ADC codes from electronics noise only (no charge from Xrays). The noise is fitted by a Gaussian function in order to determine the noise threshold. The charge-sharing region (II) represents the signal charge shared between 2 or more pixels. Region (III) is the signal region where the charge is collected in a single pixel that will be analysed to determine the CTI.

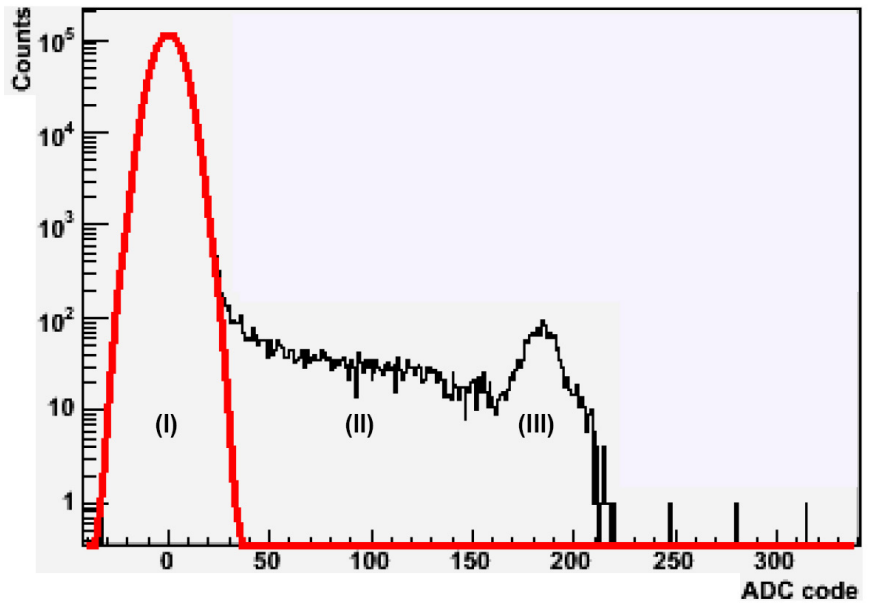

Fig. 3. Distribution of ADC codes for channel (column) two. Three regions are observed: (I) the high peak region which represents the noise, (II) the region separating the two peaks which represents the charge sharing between pixels, and (III) the X-ray peak region which represents the collected charge in a single pixel. The noise peak is fitted by a Gaussian function to determine the noise threshold. The data was taken at $-80{ }^{\circ} \mathrm{C}$ with $8 \mathrm{MHz}$ readout frequency.

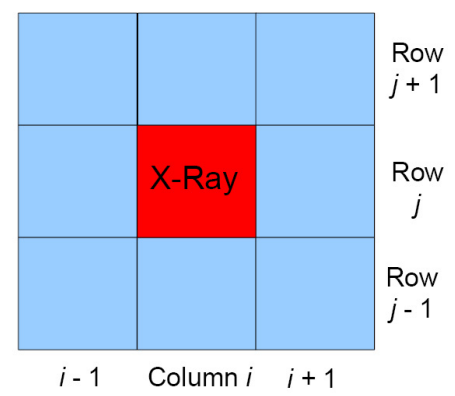

Fig. 4. Illustration of the $3 \times 3$ cluster method. A signal charge (X-ray) is only accepted if no X-ray charge is present in the neighboring pixels. The $\mathrm{X}$-ray charge is defined by its threshold determined after a first fit of the X-ray signal peak with a Gaussian function.

In order to remove hits with shared charges, a $3 \times 3$ cluster method is used (Fig. 4). This method is only applied to the 3 adjacent columns (1,2 and 3$)$. Column 2 is considered, where the signal charge of a pixel is accepted only if no X-ray charge is present in the neighboring pixels.

The noise threshold is used to separate noise from shared $\mathrm{X}$-ray charges. It is determined by fitting the noise peak with a Gaussian function. The threshold is usually set to be $5 \sigma$ above the fitted peak centroid. After removal of the noise and reduction of the charge-sharing events, a tail remains for the $\mathrm{X}$-ray peak as shown in Fig. 5 . In order to separate the X-ray signal region from the charge-sharing region we exclude all ADC codes below an X-ray threshold which is determined by fitting the X-ray peak. We usually set the threshold to be $2 \sigma$ below the fitted peak centroid but with weaker X-ray peaks the fitted Gaussian sometimes failed to follow the real shape of the peak, and then the limits were set by visual inspection. In this way we obtain the measured X-ray signal data in the pixels with full X-ray charge (Fig. 6. 


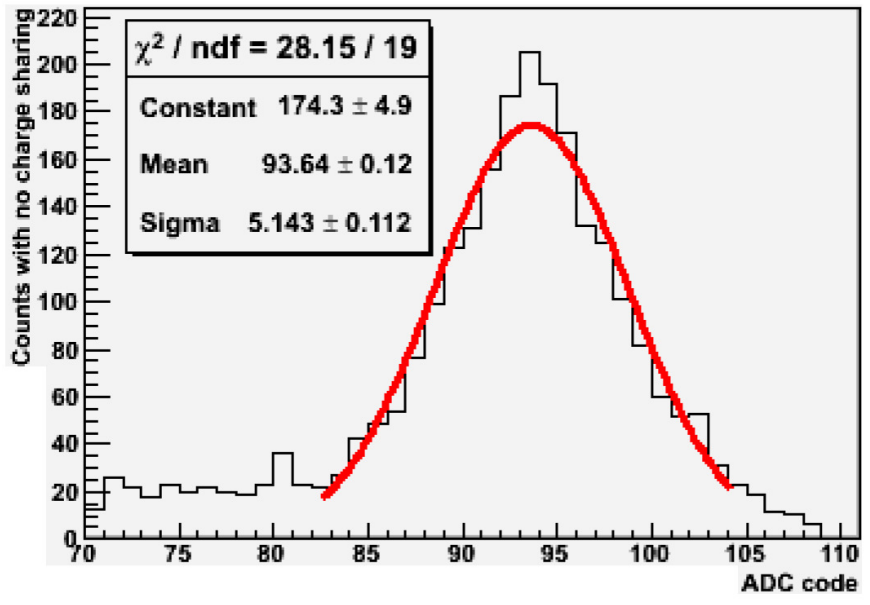

Fig. 5. X-ray signal peak with a tail from some charge sharing even after applying the $3 \times 3$ cluster method. The distribution is fitted with a Gaussian function to determine the threshold to be used to completely remove the charge sharing. This is a different dataset than that in Fig. 3 which was obtained using a higher preamplifier gain.

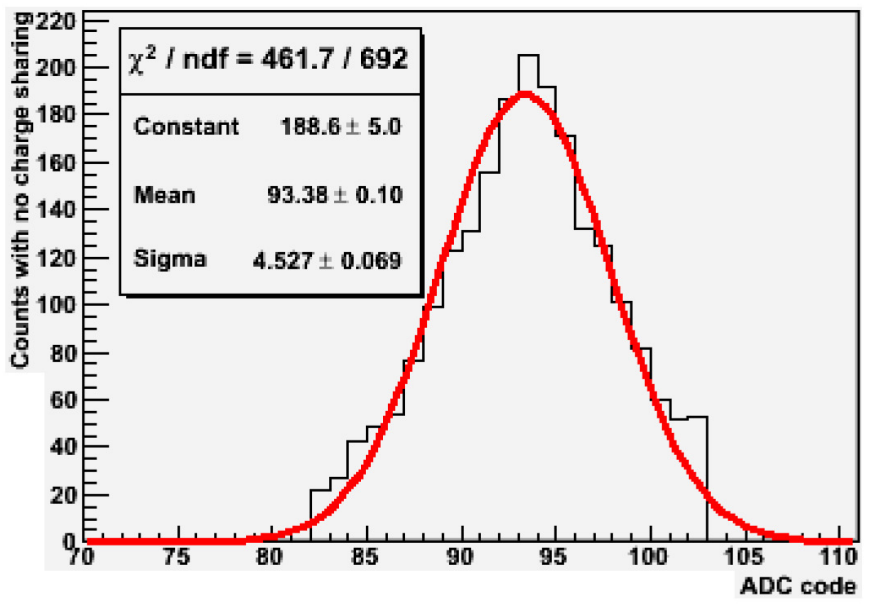

Fig. 6. X-ray signal peak with no charge sharing.

\section{BASELINE CORRECTION FOR DIFFERENT OPERATING TEMPERATURES AND READOUT FREQUENCIES}

Owing to an RC coupling at the preamplifier input, the output voltage decays exponentially with a time constant equal to $R C\left(\tau_{\mathrm{RC}}\right.$ is of the order of $\left.100 \mu \mathrm{s}\right){ }^{1}$. This is expected to give rise to an exponential baseline that varies during the clocking sequence, adding a contribution to the readout signal that depends on the pixel number. The shape of the baseline can be determined by comparing the average output level in the pixels to each other. For each pixel an average of the ADC codes was computed excluding the frames were the code exceeded the noise threshold. The average ADC code can usually be expected to be an exponential function of the pixel number with the RC decay. Figures 7, 8, 9 and 10

${ }^{1}$ For a readout frequency of $4 \mathrm{MHz}$ reading out the whole column of 400 pixels takes a time comparable to $\tau_{\mathrm{RC}}$.

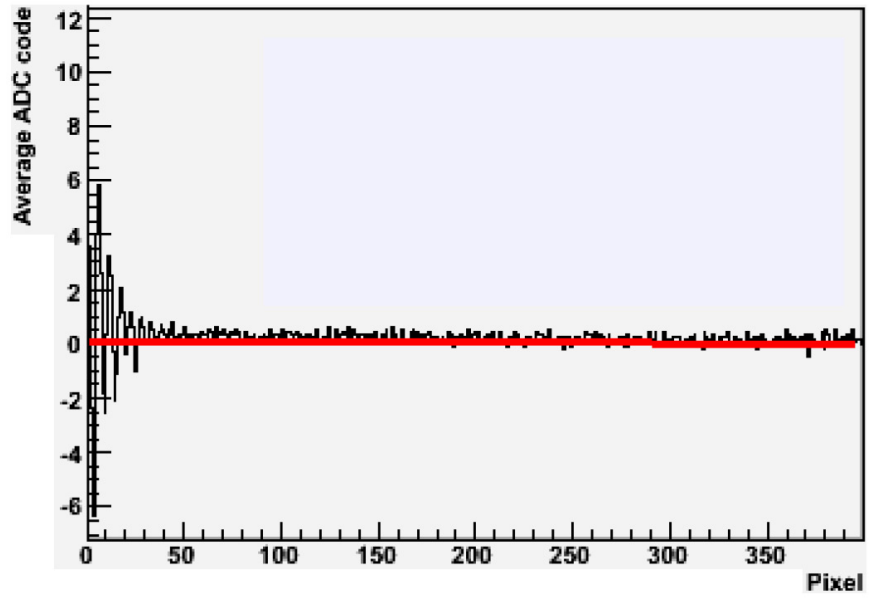

Fig. 7. Distribution of average ADC code in the noise region versus the pixel number at $2 \mathrm{MHz}$ and $-60{ }^{\circ} \mathrm{C}$ with an exponential fit. The noise region was delimited by a $3 \sigma$ noise threshold. Fit: amplitude $A=0.117 \pm 0.056$ and exponent $B=(-5.29 \pm 2.85) \times 10^{-3}$.

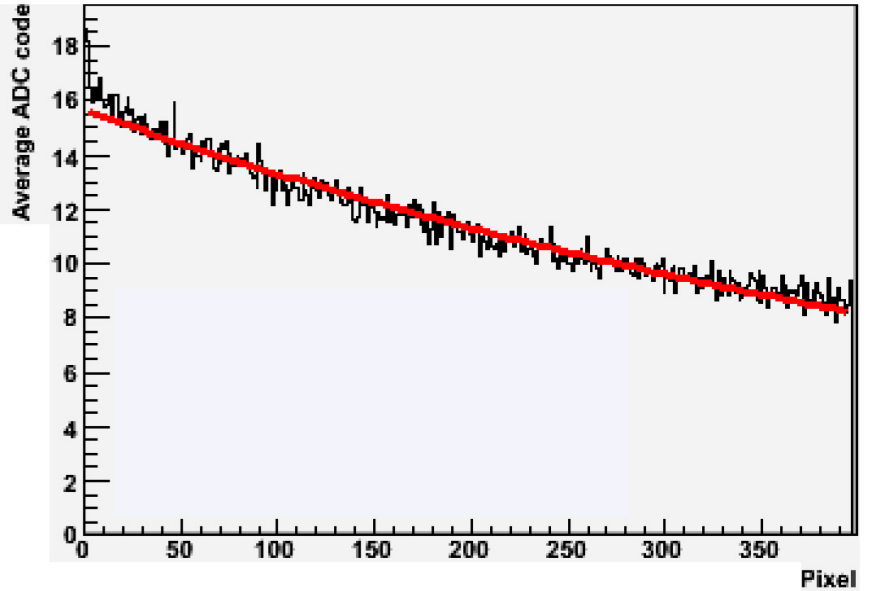

Fig. 8. Distribution of average ADC code in the noise region versus the pixel number at $4 \mathrm{MHz}$ and $-6{ }^{\circ} \mathrm{C}$ with an exponential fit. The noise region was delimited by a $3 \sigma$ noise threshold. Fit: amplitude $A=15.64 \pm 0.44$ and exponent $B=(-16.24 \pm 1.33) \times 10^{-4}$.

show the distributions of the average ADC codes for all frames of a measurement as a function of the pixel number for different readout frequencies and operating temperatures. These distributions are fitted by an exponential function given by $A \exp (-B j)$, where $A$ corresponds to the signal charge at the first pixel, $B$ is the slope and $j$ is the pixel number. This function will be subtracted from the X-ray data. Figures 7 and 10 show approximately zero baseline level, whereas Figs. 8 and 9 show non-zero baseline fits. The baseline level is much smaller at low temperatures, for example at $-60{ }^{\circ} \mathrm{C}$ (Fig. 7) and at $-109{ }^{\circ} \mathrm{C}$ (Fig. 10). Figures 8 and 9 show baselines for higher temperatures $-6{ }^{\circ} \mathrm{C}$ and $-17^{\circ} \mathrm{C}$, respectively. The observed baselines show different frequency and temperature dependence from what is expected from the $\mathrm{RC}$ effect. This indicates that other electronic effects also influence the baseline. 


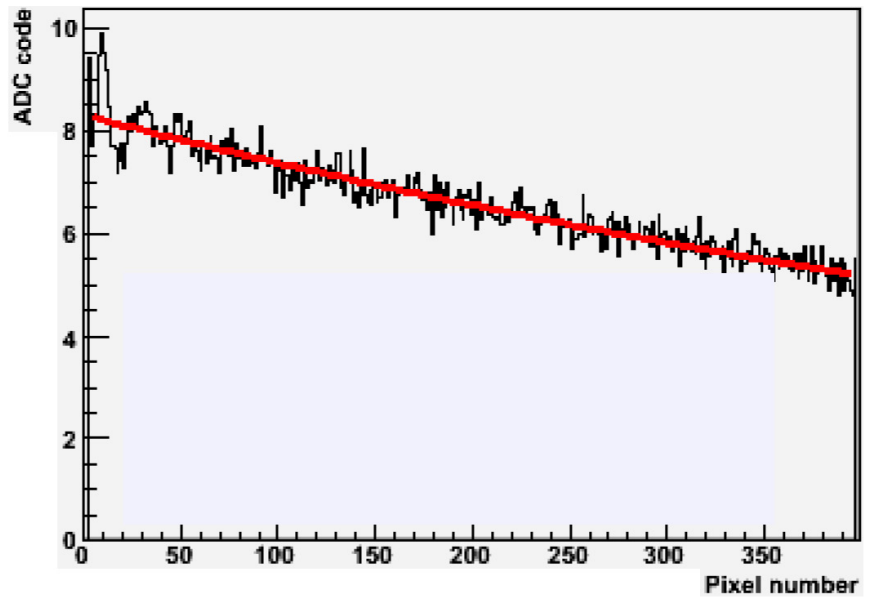

Fig. 9. Distribution of average ADC code in the noise region versus the pixel number at $8 \mathrm{MHz}$ and $-17^{\circ} \mathrm{C}$ with an exponential fit. The noise region was delimited by a $3 \sigma$ noise threshold. Fit: amplitude $A=8.31 \pm 0.31$ and exponent $B=(-11.86 \pm 1.74) \times 10^{-4}$.

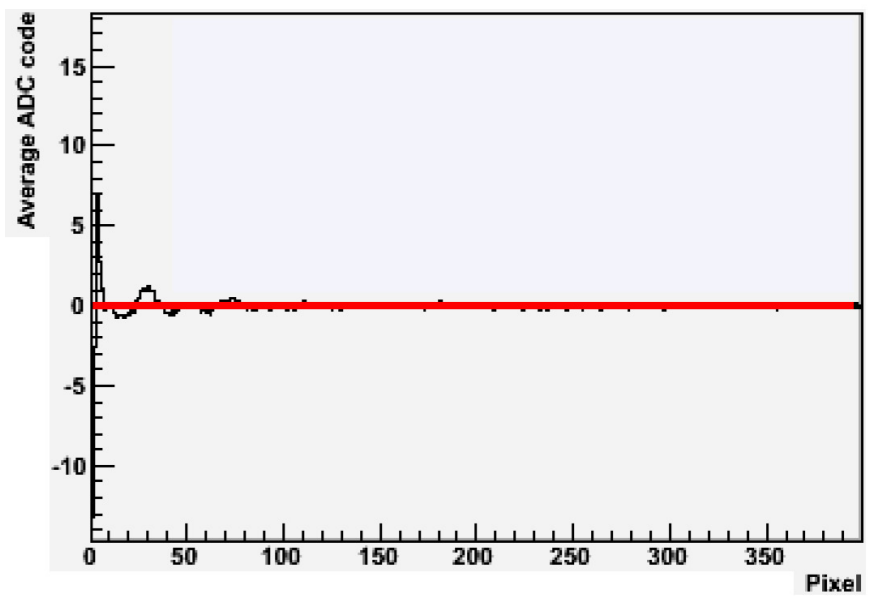

Fig. 10. Distribution of average ADC code in the noise region versus the pixel number at $8 \mathrm{MHz}$ and $-109{ }^{\circ} \mathrm{C}$ with an exponential fit. The noise region was delimited by a $3 \sigma$ noise threshold. Fit: amplitude $A=(0.082 \pm$ $5.035) \times 10^{-3}$ and exponent $B=(1.073 \pm 16.853) \times 10^{-2}$

\section{Determination of Charge Transfer INEFFICIENCY}

The charge transfer inefficiency (CTI) in one pixel is defined as the ratio of signal lost during transfer (captured by traps) to the initial signal charge. For an un-irradiated CCD we expect that the CTI value is consistent with zero within uncertainties. In order to determine the CTI we first make the overclock correction and apply the $3 \times 3$ cluster method. Then, the X-ray peak was fitted with the Gaussian function to determine the $\mathrm{X}$ ray threshold. This was used to construct the two distributions with a fit of average ADC codes as shown in Fig. 11 and with a fit of individual events (X-ray hits) as shown in Fig. 12. Both distributions are fitted with the first-order polynomial function $P_{0}+P_{1} j$, where $P_{0}$ corresponds to the charge at the first pixel, $P_{1}$ is the slope and $j$ is the pixel number. These distributions are plotted without baseline removal. Figures 13 and 14 show the distributions of the averages and all events as a function of pixel number with baseline removal. The CTI is determined for the four cases using $C T I=-P_{1} / P_{0}$.

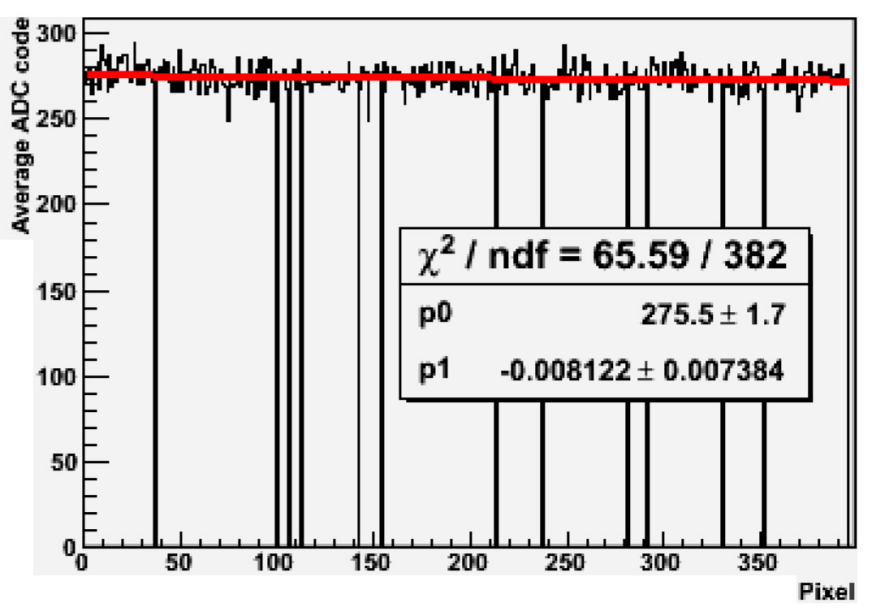

Fig. 11. Fit to average ADC codes without baseline removal at $2 \mathrm{MHz}$ and $-31^{\circ} \mathrm{C} . C T I=(2.99 \pm 2.69) \times 10^{-5}$.

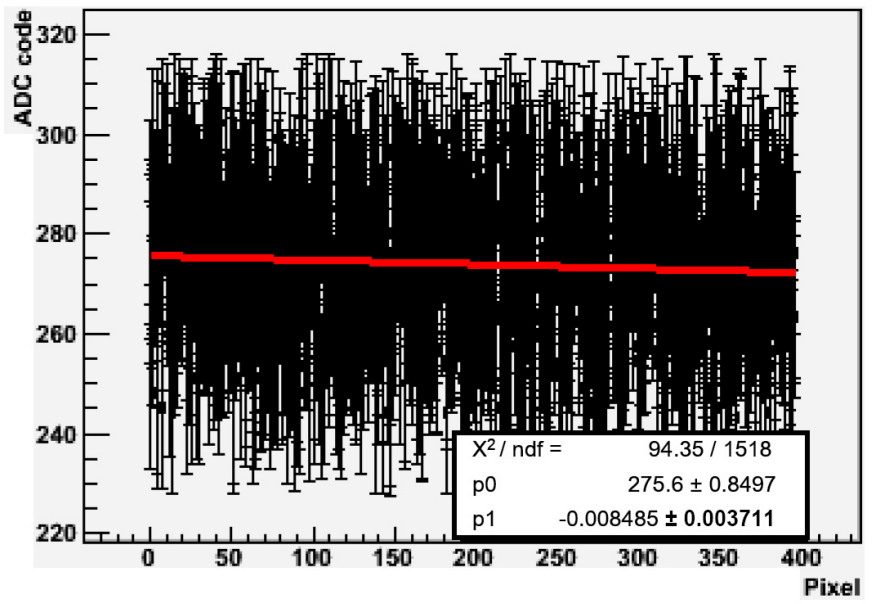

Fig. 12. Fit to events (hits) without baseline removal at $2 \mathrm{MHz}$ and $-31^{\circ} \mathrm{C}$ $C T I=(3.69 \pm 1.31) \times 10^{-5}$.

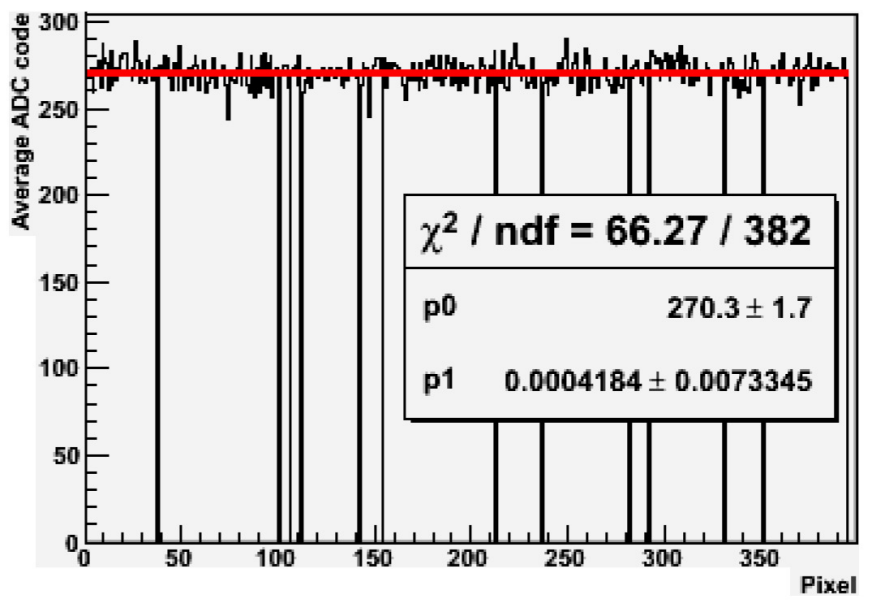

Fig. 13. Fit to average ADC codes with baseline removal at $2 \mathrm{MHz}$ and $-31{ }^{\circ} \mathrm{C} . C T I=(-0.09 \pm 2.70) \times 10^{-5}$. 


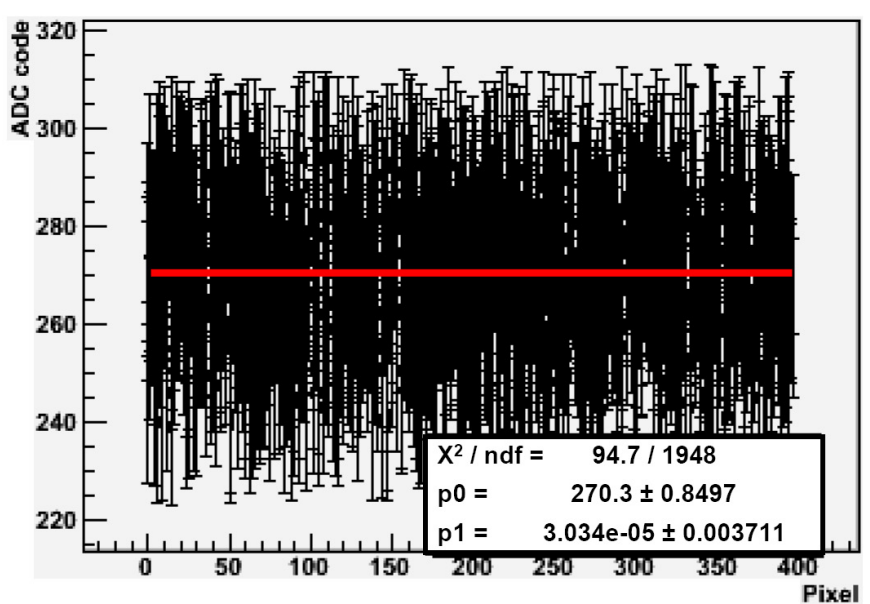

Fig. 14. Fit to events (hits) with baseline removal at $2 \mathrm{MHz}$ and $-31{ }^{\circ} \mathrm{C}$. $C T I=(0.63 \pm 1.33) \times 10^{-5}$.

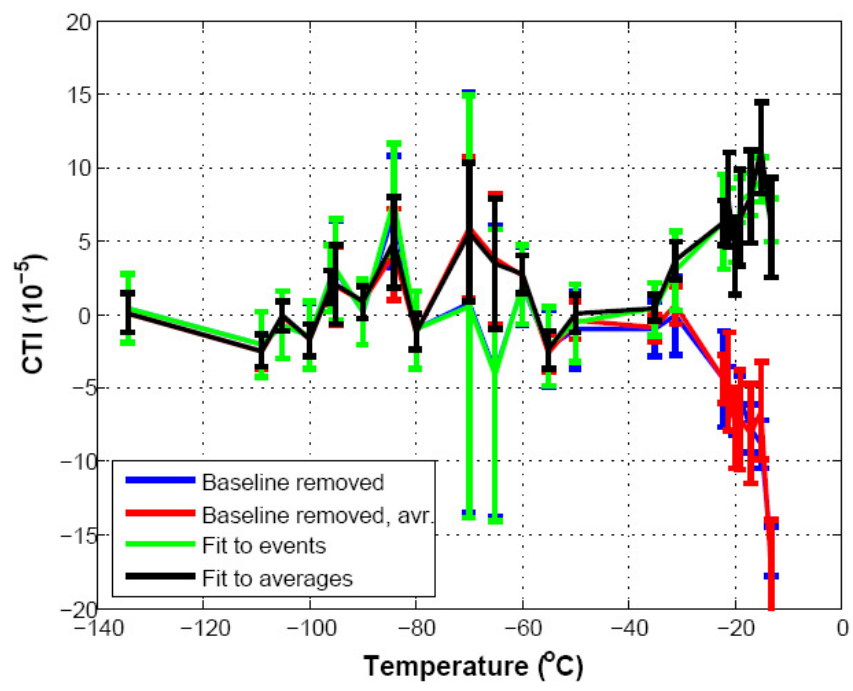

Fig. 15. CTI versus temperature at $2 \mathrm{MHz}$ readout frequency. The CTI is shown resulting from fits to average ADC codes and to events (hits), with and without baseline removal. The shown error bars result from the precision of the fits.

\section{CTI RESULTS PRE-IRRADIATION FOR DIFFERENT READOUT FREQUENCIES}

Figures 15, 16 and 17 show the CTI values as a function of temperature for an un-irradiated CPC1 at different readout frequencies. The CTI has been calculated using two methods, a linear fit of averages versus pixel number and using a linear fit of events (X-ray hits) versus pixel number. As it is expected for an un-irradiated CCD, the CTI is small because the density of traps is small. In the temperature region below $-25{ }^{\circ} \mathrm{C}$ no frequency dependence of the CTI measurements is observed. One may note that in the region of rather high temperatures (above $-25{ }^{\circ} \mathrm{C}$ ) the effect of baseline removal is large and the determined CTI values indicate that the baseline effect is overestimated. The effect of the baseline removal decreases with increasing frequency as the readout clocking sequence becomes shorter compared to $\tau_{\mathrm{RC}}$ (as visible in Fig. 17 for $8 \mathrm{MHz}$ readout frequency).

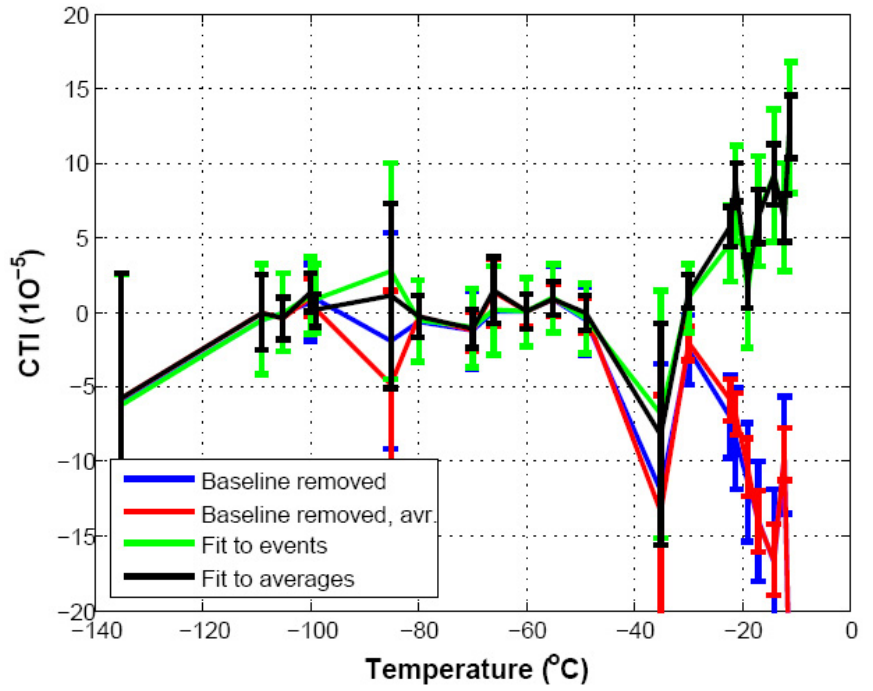

Fig. 16. CTI versus temperature at $4 \mathrm{MHz}$ readout frequency. The CTI is shown resulting from fits to average ADC codes and to events (hits), with and without baseline removal. The shown error bars result from the precision of the fits.

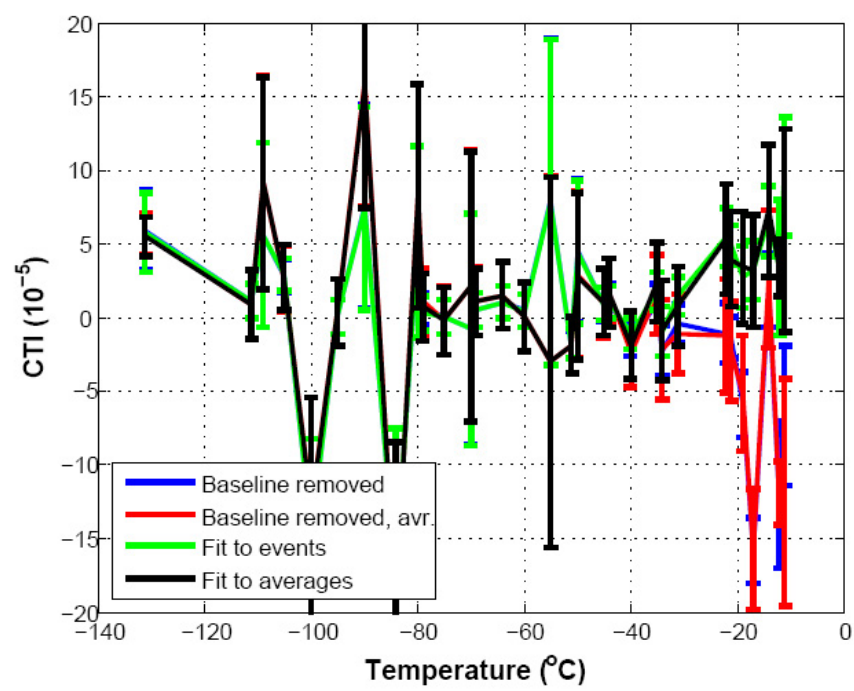

Fig. 17. CTI versus temperature at $8 \mathrm{MHz}$ readout frequency. The CTI is shown resulting from fits to average ADC codes and to events (hits), with and without baseline removal. The shown error bars result from the precision of the fits.

\section{CONCLUSIONS AND OUTLOOK}

An un-irradiated CPCCD is operated in a range of temperatures from $-10{ }^{\circ} \mathrm{C}$ to $-136{ }^{\circ} \mathrm{C}$ (liquid nitrogen cooling) with different readout frequencies 2,4 and $8 \mathrm{MHz}$. The spectrum of a ${ }^{55} \mathrm{Fe}$ source is measured with this device. The CTI is analysed for different readout frequencies and operating temperatures. A clear X-ray signal is extracted by identifying isolated hits $(3 \times 3$ method $)$. The baseline is subtracted. The CTI value is small and compatible with zero as can be expected for an un-irradiated CPCCD. Further CTI measurements with a CPCCD after irradiation and refinement of the analysis method are planned. 


\section{ACKNOWLEDGEMENTS}

We would like to thank Salim Aoulmit, Alex Chilingarov and Lakhdar Dehimi for discussions and comments on the manuscript. This work is supported by the Science and Technology Facilities Council (STFC) and Lancaster University. $\mathrm{KB}$ wishes to thank the Algerian Government for financial support and Lancaster University for their hospitality. AS would like to thank the Faculty of Science and Technology at Lancaster University for financial support and the organizers of the IEEE`08 conference for their hospitality.

\section{REFERENCES}

[1] C.J.S. Damerell, "Radiation damage in CCDs used as particle detectors", ICFA Instrum. Bull. 14 (1997) 1.

[2] K. Stefanov, PhD thesis, Saga University (Japan), "Radiation damage effects in CCD sensors for tracking applications in high energy physics", 2001, and references therein; K. Stefanov et al., "Electron and neutron radiation damage effects on a two phase CCD", IEEE Trans. Nucl. Sci. 47 (2000) 1280

[3] LCFI collaboration homepage: http://hepwww.rl.ac.uk/lcfi/

[4] S.D. Worm, "Recent CCD development for the vertex detector of the ILC - Including ISIS (In-situ Storage Image Sensors)", in 10th Topical Seminar on Innovative Particle and Radiation Detectors (IPRD06), Siena, Italy, October 1-5, 2006.

[5] T.J. Greenshaw, "Column Parallel CCDs and in-situ storage image sensors for the vertex detector of the international linear collider", in 2006 Nuclear Science Symposium, San Diego, USA, October 29November 4, 2006.

[6] M.S. Robbins "The Radiation Damage Performance of Marconi CCDs", Marconi Technical Note S\&C 906/424 2000 (unpublished).

[7] J.E. Brau and N.B. Sinev, "Operation of a CCD particle detector in the presence of bulk neutron damage", IEEE Trans. Nucl. Sci. 47 (2000) 1898.
[8] J.E. Brau, O. Igonkina, C.T. Potter and N.B. Sinev, "Investigation of radiation damage effects in neutron irradiated CCD", Nucl. Instr. and Meth. A549 (2005) 1173.

[9] A.M. Mohsen and M.F. Tompsett, "The effect of bulk traps on the performance of bulk channel charge-coupled devices", IEEE Trans. Electron Dev. ED21, 11 (1974) 701.

[10] I.H. Hopkins, G. Hopkinson and B. Johlander, "Proton-induced charge transfer degradation in CCD's for near-room temperature applications", IEEE Trans. Nucl. Sci. 41 (1994) 1984.

[11] T. Hardy, R. Murowinski and M.J. Deen, "Charge transfer efficiency in proton damaged CCD's”, IEEE Trans. Nucl. Sci. 45 (1998) 154.

[12] A. Sopczak, "LCFI Charge Transfer Inefficiency Studies for CCD Vertex Detectors", IEEE 2005 Nuclear Science Symposium, San Juan, USA Proc. IEEE Nuclear Science Symposium Conference Record N37-7 (2005) 1494.

[13] A. Sopczak, "Radiation Hardness of CCD Vertex Detectors for the ILC", IEEE 2006 Nuclear Science Symposium, San Diego, USA. October 29November 4, 2006. Proc. IEEE Nuclear Science Conference Record N14-215 (2006) 576.

[14] A. Sopczak et al., "Simulations of the Temperature Dependence of the Charge Transfer Inefficiency in a High-Speed CCD", IEEE Trans. Nucl. Sci. 54 (2007) 1429, and references therein.

[15] A. Sopczak et al., "Radiation Hardness Studies in a CCD with HighSpeed Column Parallel Readout", Proc. 2007 IEEE Nuclear Science Symposium, October 27-November 3, 2007, Honolulu, USA. Proc. IEEE Nuclear Science Conference Record N48-2 (2007) 2278.

[16] A. Sopczak et al., "Radiation Hardness Studies in a CCD with Highspeed Column Parallel Readout", JINST 3 (2008) 5007.

[17] J.W. Walker and C.T. Sah, " Properties of 1.0-MeV-electron-irradiated defect centers in silicon", Phys. Rev. B7 (1972) 4587.

[18] N.S. Saks, "Investigation of Bulk Electron Traps Created by Fast Neutron Irradiation in a Buried N-Channel CCD", IEEE Trans. Nucl. Sci. 24 (1977) 2153.

[19] J.R. Srour, R.A. Hartmann and S. Othmer, "Transient and Permanent Effects of Neutron Bombardment on a Commercially Available NBuried-Channel CCD”, IEEE Trans. Nucl. Sci. 27 (1980) 1402

[20] ROOT homepage: http://root.cern.ch/ 